\title{
Discussion: Fuzzy logic model to predict hydraulic jump aeration efficiency
}

\section{S. Kucukali and S. Cokgor}

\section{Contribution by A. Altunkaynak, Istanbul Technical University, Turkey \\ In this paper the authors developed a fuzzy logic model to predict hydraulic jump aeration efficiency but parts of the paper appear to be ambiguous, there are points that may need further clarification in both the application of methodology and the results sections.}

First, the most important step in developing a fuzzy logic model, which is a kind of blackbox model, is to train and test the model properly. For these operations, the available data are normally split into two parts. While approximately two-thirds of the total data are used for training, the rest of the data are left for testing. The authors of the paper do not appear to have followed this procedure. It may have been better if they had used the training data to determine the fuzzy rule based membership functions of model inputs ( $F r 1$ and $R e$ ) and output by considering the least prediction errors and then validated the model using an independent data set such as test data. The absence of these procedures makes the results of the study questionable because it is a well known fact that validating a model with the data that are used at the same time for establishing the model produces high correlations coefficients between observed and predicted values.

The authors say that they used a trial and error approach to determine the membership functions, but they failed to explain how this was done. The authors refer to some papers in the literature which explain this issue in detail (Altunkaynak et al., 2005a; Altunkaynak et al., 2005b; Şen and Altunkaynak, 2004).

Second, the number of rules in the fuzzy rule base theoretically should be $5 \times 5 \times 7=175$ which is the multiplication of input and output membership functions numbers. In the paper, however, the authors use only 21 rules to express the system behaviour. It would be helpful if the authors explained why they reduced the number of rules in the fuzzy rule base. In addition there is no indication of how they decided the number of input and output membership functions.

Third, although the authors give the equations of the multiple regression model, they do not compare the results with the fuzzy model results. It would be useful if such a comparison could be made.

The fuzzy logic approach is a very flexible and reliable method to determine the relationship between input and output variables, and is easy to use. The misapplication of fuzzy logic methods may, however, lead to misinterpretations of the results. This means that every step in the application should be applied carefully and properly.

\section{Authors' reply}

As the contributor stated, the data were not trained in the model, this is because there were limited experimental data and these values were used for the model validation. In further studies, however, the data sets of Avery and Novak (1978) and Wilhelms et al. (1982) could be used for the model calibration. The fuzzy logic rules were written on the basis of experimental observations and system behaviour. This issue is also known as expert decision and this enables the fuzzy logic algorithm to mimic human thinking.

In the model, upstream Froude $\left(F r_{l}\right)$ and Reynolds $(R e)$ numbers were selected as input parameters, where $F r_{\text {I }}$ is the dominant parameter. The studies demonstrated that the gas transfer efficiency of the hydraulic jump is under the control of these parameters. The Froude number was divided into five fuzzy subsets as follows: $2 \cdot 3-2 \cdot 4$ very low, $2 \cdot 4-3 \cdot 4$ low, $3-7$ medium, 4-9 high, and 6-15 very high. These fuzzy subsets and membership functions were created based on the physical behaviour of the system. For example, in the experiments, air entrainment took place at $F r_{1}>2.3$ and the influence of $F r_{l}$ strengthened at $F r_{I}>4$. Above $R e>1 \times 10^{5}$, the Reynolds number effect was not taken into account, which represents the fully turbulent conditions.

The fuzzy rules were sufficient to relate the parameters and they captured the dynamic behaviour of the system (Kucukali and Cokgor, 2007). There was no need to write 175 rules, which was proposed by the discusser, because it will make the model unpractical and complicated. The Avery and Novak formula also gave sufficient results for the experiments. What this equation suffers from is the lack of constraints of $F r_{I}$ and Re numbers as discussed above. In the proposed model, however, these physical constraints were taken into account. 


\section{REFERENCES}

Altunkaynak A, Özger M and Çakmakçı M (2005a) Water consumption prediction of Istanbul city by using fuzzy logic approach. Water Resources Management 19(5): 641-654.

Altunkaynak A, Özger M and Çakmakçı M (2005b) Fuzzy logic modeling of the dissolved oxygen fluctuations in Golden Horn. Ecological Modeling 189(3-4): 436-446.

Avery ST and Novak P (1978) Oxygen transfer at hydraulic structures. Journal of Hydraulic Engineering. ASCE 104(11): $1521-1540$.
Kucukali S and Cokgor S (2007) Fuzzy logic model to predict hydraulic jump aeration efficiency. Proceedings of the Institution of Civil Engineers, Water Management 160(4): $225-231$.

Şen Z and Altunkaynak A (2004) Fuzzy logic in rainfall and runoff modeling. Nordic Hydrology 35(1): 31-43.

Wilhelms SC, Clark L, Wallace JR and Smith DR (1982) Gas Transfer in Hydraulic Jumps. US Army Engineer Waterways Experiment Station, Vicksburg, MS, USA, Technical Report E-81-10.

\section{What do you think?}

To discuss this paper, please email up to 500 words to the editor at journals@ice.org.uk. Your contribution will be forwarded to the author(s) for a reply and, if considered appropriate by the editorial panel, will be published as discussion in a future issue of the journal.

Proceedings journals rely entirely on contributions sent in by civil engineering professionals, academics and students. Papers should be 2000-5000 words long (briefing papers should be 1000-2000 words long), with adequate illustrations and references. You can submit your paper online via www.icevirtuallibrary.com/content/journals, where you will also find detailed author guidelines. 